\title{
GPR 109A alleviate mastitis and enhances the blood milk barrier by activating AMPK/Nrf2 and autophagy
}

\author{
Wenjin Guo, Wen Li, Yingchun Su, Shu Liu, Xingchi Kan, Xin Ran, Yu Cao, Shoupeng Fu ${ }^{\bowtie}$, Juxiong Liu ${ }^{\bowtie}$ \\ College of Veterinary Medicine, Jilin University, Changchun 130062, China. \\ $\bowtie$ Corresponding authors: E-mail: fushoupeng@jlu.edu.cn (S.F.); juxiong@jlu.edu.cn (J.L).
}

(C) The author(s). This is an open access article distributed under the terms of the Creative Commons Attribution License (https://creativecommons.org/licenses/by/4.0/). See http://ivyspring.com/terms for full terms and conditions.

Received: 2021.05.09; Accepted: 2021.10.04; Published: 2021.10.17

\begin{abstract}
Mastitis causes great psychological and physical pain among women. Our previous studies found that niacin has anti-inflammatory effect, and the realization of this function depends on GPR109A. However, there are no previous reports about the anti-inflammatory function of GPR 109A in mastitis. In our study, we observed the effect of niacin on the WT and GPR 109A- mice mastitis model. The results showed that administration of niacin to WT mice reduced the damage, proinflammatory mediators and protected the integrity of the blood milk barrier in mammary gland. While in GPR $109 \mathrm{~A}^{-\%}$ mice, there was no effect on the above indexes. In mammary epithelial cells, GPR109A was able to promote autophagy and Nrf2 nuclear import through AMPK. In LPS-induced mammary epithelial cells, niacin inhibited the LPS-induced inflammatory response and downregulation of tight junction proteins, and these effects were eliminated by knocking down GPR 109A, blocking autophagy or inhibiting Nrf2 nuclear import. These results indicate that in mastitis, GPR109A promotes autophagy and Nrf2 nuclear import through AMPK, thereby inhibiting inflammatory damage to the mammary gland and repairing the blood milk barrier. Our results suggested that GPR109A may be a potential target for the treatment of mastitis.
\end{abstract}

Key words: Niacin; Mastitis; GPR109A; AMPK/Nrf2; Autophagy; Blood milk barrier

\section{Introduction}

Mastitis often occurs during lactation in women [1]. Especially for the women who were give birth to a child for the first time, the incidence rate of mastitis in lactation was as high as $2-4 \%$ [2]. When mastitis occurs in the breast, it causes local redness, swelling, pain and other symptoms [3]. If not treated in time, mastitis can cause suppuration and septicemia, which endanger the life of affected women [4]. Mastitis causes great physical and psychological pain, and the more advanced the disease, the stronger the pain [5]. Therefore, timely prevention and control of mastitis can effectively reduce pain in women.

At present, the problem of how to most effectively treat mastitis in women in underdeveloped areas has not been solved, and mastitis still seriously affects the health of women in underdeveloped areas. Some studies have shown that mastitis can increase the risk of HIV transmission, especially in underdeveloped areas [6]. Some studies have shown that women's reproductive health was affected by mastitis, so understanding mastitis and developing new treatments are of great importance.

The blood milk barrier formed by breast during lactation is an important barrier for the body [7], and it is closely related to the health of lactating women [8]. Tight junction is an important part of the formation of blood milk barrier [9]. Mastitis can severely damage the blood milk barrier [10]. Wang Jingjing showed that mastitis caused by LPS can also seriously damage the blood milk barrier and aggravate inflammation [11]. These studies have shown that breaking the blood milk barrier will lead to the aggravation of mastitis, and the aggravation of mastitis will also affect the blood milk barrier in turn. Therefore, how to protect the blood milk barrier and reduce the inflammatory reaction in the mammary gland is of positive significance to alleviate mastitis.

Niacin belongs to $\mathrm{B}$ vitamins and exists in animals and plants. And niacin is one of the 13 essential vitamins for the human body. Some studies 
reported that niacin can alleviate inflammatory response. Some studies have shown that niacin can reduce pulmonary hypertension through H-PGDs in macrophages, and some studies have shown that niacin can protect blood vessels by activating HO-1 in vivo and in vitro $[12,13]$. These studies suggested that niacin may play an anti-inflammatory role in different parts of the body.

GPR109A is a G-protein-coupled receptor that regulates cellular activities by sensing various extracellular signals [14]. Some studies have shown that GPR109A can alleviate TNBS-induced colitis by inhibiting p65 nuclear import [15]. Other studies have shown that GPR109A can reduce the inflammatory response of microglia, thereby protecting neurons and alleviating Parkinson's symptoms in mice [16]. These studies indicate that GPR109A has a good anti-inflammatory function and is expressed in many kinds of cells and tissues. In addition, GPR109A is highly expressed in the mammary gland [17]. However, the anti-inflammatory function of GPR109A in the mammary gland has not been reported previously.

Nrf2 plays an important role in redox reaction $[18,19]$. Nrf2 can reduce cell damage caused by oxidative stress and maintain cell homeostasis [20,21]. Previous studies have shown that insufficient niacin intake significantly reduces $\mathrm{Nrf} 2$ expression and increases the expression of proinflammatory mediators in young grass carp [22]. However, there are no available reports on the activation of Nrf2 by niacin in mammals.

Autophagy is an important means for cells to maintain normal life activities, and autophagy is involved in various inflammatory reactions and barrier repair [23,24]. MiR-155 can alleviate lung injury by regulating autophagy [25]. It has also been shown that induction of autophagy can alter tightly linked scaffold proteins [26]. So autophagy is closely related to inflammation and the blood milk barrier, but there are few studies on the role of autophagy in mastitis or its role in maintaining the blood milk barrier. Therefore, we speculate that GPR109A may alleviate inflammation and enhance the blood milk barrier by activating autophagy and promoting Nrf2 nuclear import.

\section{Materials and methods}

\section{Reagents}

3-methyladenine (3-MA) and Dorsomorphin (Compound C, CC) were supported from Selleckchem (Shanghai, China). ML385 was purchased from MedChemExpress (USA). Niacin was purchased from sigma (USA).

\section{Animal experiments}

In our experiments, All GPR109A-/- and wildtype (WT) pregnant female mice $(\mathrm{C} 57 \mathrm{BL} / 6)$ were 9-week-old, The GPR109A $\%$ mice is a generous gift from Dr. Martin Sager in Germany. The experiments were approved by the Jilin University Institutional Animal Care and Use Committee.

The whole animal experiment was divided into 8 groups. WT mice included NT group, niacin group, LPS group and niacin + LPS group, and GPR109A \%mice also included the above four groups. Then prepare purified water containing $50 \mathrm{mM}$ niacin to ensure the freedom of diet and drinking water for mice. At the beginning of the experiment, the niacin group and niacin + LPS group in WT and GPR109A-/mice were fed with purified water containing niacin until the end of the experiment. WT mice and GPR109A /- were caged respectively. One male mouse and two female mice were caged for one week. After pregnancy, the female rats were divided into groups and fed purified water containing niacin. The pregnancy cycle of mice was 21 days, and the experiment was carried out 6 days after birth. Therefore, the whole niacin feeding cycle was 27 days. On day 26, the mice were anesthetized and LPS was injected into the fourth pair of inguinal mammary glands of the mice. The mice were then put back into the cage and the mammary glands were collected 24 hours later. During this period, they are free to eat and drink water, and the drinking water is purified water containing niacin. The suckling mice were fed to other female mice.

\section{Protein levels of MDA, TNF- $\alpha$, IL-6 and IL-1 $\beta$}

Firstly, the mouse mammary gland tissue was ground and the supernatant was collected, and then the contents of TNF- $\alpha$, IL- 6 and IL-1 $\beta$ in the supernatant were detected. All detection steps refer to the instructions in the ELISA kit (CA, Biolegend, Cat: 430904, Cat: 431304, Cat: 432604), and the addition amount of supernatant is adjusted according to the actual situation.

\section{Myeloperoxidase (MPO) activity assay}

The collected fresh mouse mammary glands were ground and the supernatant was collected. MPO content in mouse mammary gland was detected through the previous test steps of our research group [27].

\section{Evaluation of histological changes}

H\&E staining procedure and pathological score in this study were according to previously described [28]. 


\section{Construction of GPR 109A knockdown plasmid}

The construction of GPR109A knockdown plasmid (GPR109A-shRNA) was carried out by Songon Biotech (Shanghai, China). Four sequences of GPR109A knockdown plasmids were designed. We found that the knockdown effect of shRNA1 was the best among these plasmids through pre-experiment (S 2).

\section{Cell culture}

EpH4-Ev cells were cultured in DMEM medium (Gibco, Grand Island, NY, USA) containing 10\% fetal bovine serum (Hyclone, USA) at $37^{\circ} \mathrm{C}$ in a humidified incubator with $5 \% \mathrm{CO}_{2}$.

\section{The effect of niacin on Eph4-Ev activity was detected by Cell counting kit-8 assay}

Eph4-Ev cells were inoculated into 96 well plates and added $100 \mu \mathrm{L}$ culture medium. After stimulation with LPS or niacin for 24 hours, CCK-8 reagent was added to the well which inoculated with cells, and the proportion of addition refers to the instructions. After waiting for 1 hour, detect the absorbance with $450 \mathrm{~nm}$ wavelength.

\section{Detection of barrier permeability by FITC-albumin}

To verify the integrity of the blood milk barrier, fresh mammary gland tissue was immersed in PBS containing FITC-albumin. After 20 minutes, the mammary gland tissue was immersed in liquid nitrogen, and the frozen section was performed. Then the green fluorescence in the section was observed under fluorescence microscope. The detailed steps refer to the previous protocol [11].

\section{The mRNA level in mammary gland tissue or mammary epithelial cells was detected by qRT-PCR}

We added TRIzol to collected mammary tissue or Eph4-EVs. Then, the total RNA in mammary tissue or Eph4-EVs was collected by a series of treatments such as chloroform, isopropanol and alcohol. Finally, the collected RNA was reverse transcribed into cDNA. The experimental method of reverse transcription was descripted previously [27]. Three independent operations were performed in each experiment. The primers we designed are shown in Table 1.

\section{Immunofluorescence}

We quickly frozen the fresh mammary tissue in liquid nitrogen, then treated the tissue block with OCT, and put it into a frozen slicer for slicing after OCT solidification. The frozen sections were stained with immunofluorescence according to the previous steps [27]. The primary antibodies used in this study were diluted as follows: anti-Nrf2 (1:150), antiClaudin 3 (1:200), anti-ZO-1 (1:200) and anti-Occludin (1:200). All immunofluorescence staining will be observed and photographed by fluorescence microscope.

Table 1. Primer sequences of TNF- $\alpha$, iNOS, IL-6, IL-I $\beta$ and COX-2.

\begin{tabular}{|c|c|c|c|}
\hline Item & Primer & $\begin{array}{l}\text { Length } \\
\text { (bp) }\end{array}$ & $\begin{array}{l}\text { Accession } \\
\text { number }\end{array}$ \\
\hline IL-6 (sense) & $\begin{array}{l}\text { 5'-CCGGAGAGGAGACTTCA } \\
\text { CAG-3' }\end{array}$ & 134 & NM_031168 \\
\hline IL-6 (anti-sense) & 5'- CAGAATTGCCATTGCACAAC-3' & & \\
\hline$T N F-a$ (sense) & 5'-ACGGCATGGATCTCAAAGAC-3' & 116 & NM_013693 \\
\hline TNF- $a$ (anti-sense) & 5'-GTGGGTGAGGAGCACGTAGT-3' & & \\
\hline$I L-1 \beta$ (sense) & 5'-GCTGCTTCCAAACCTTTGAC-3' & 121 & NM_008361 \\
\hline$I L-1 \beta$ (anti-sense) & 5'-AGCTTCTCCACAGCCACAAT-3' & & \\
\hline COX-2 (sense) & 5'-AGAGTCAGTTAGTGGGTAGT-3' & 170 & NM_011198 \\
\hline COX-2 (anti-sense) & $\begin{array}{l}\text { 5'-CTTGTAGTAGGCTTAAACA } \\
\text { TAG-3' }\end{array}$ & & \\
\hline$i N O S$ (sense) & $\begin{array}{l}5^{\prime} \text {-TCAGACCAAGATCAAGAGCGT } \\
\text { GTTG-3' }\end{array}$ & 128 & NM_010927 \\
\hline iNOS (anti-sense) & $\begin{array}{l}\text { 5'-GGAACTGTTGAGGAGCGGACA } \\
\text { AG-3' }\end{array}$ & & \\
\hline$\beta$-actin (sense) & $\begin{array}{l}\text { 5'-GTCAGGTCATCACTATCGGC } \\
\text { AAT-3' }\end{array}$ & 147 & NM_007393 \\
\hline$\beta$-actin (anti-sense) & $\begin{array}{l}\text { 5'-AGAGGTCTTTACGGATGTCAA } \\
\text { CGT-3' }\end{array}$ & & \\
\hline
\end{tabular}

\section{Electron microscopy}

The operation procedure and method of TEM were all referred to the previous description [29].

\section{Plasmids and fluorescence microscopy}

Plasmid of mRFP-GFP-LC3 was transfected into the EpH4-Evs by LipoFiter (HANBIO), and then observed by laser confocal microscope.

\section{Co-immunoprecipitation}

We first collected the total protein of Eph4-ev cells, and then operated according to the instructions of Pierce ${ }^{\mathrm{TM}}$ Classic Magnetic IP/Co-IP Kit (Thermo Fisher, USA).

\section{The protein was detected by Western blotting}

Firstly, mammary tissue or Eph4-EVs were lysed with protein lysate to collect total protein. Then Pierce BCA Protein Assay Kit (Thermo Fisher, USA) was used to detect the concentration of the extracted total protein, add appropriate $5 \times \mathrm{SDS}$ according to the protein concentration, and finally boil for 5 minutes to denature the protein. Western blotting was performed using standard protocols. The primary antibodies used are shown in Table 2.

\section{Statistical analysis}

All images and analyses were performed using GraphPad Prism software (La Jolla, CA, USA). All data were expressed as mean $\pm \mathrm{SD}$. When the 
grouping is greater than or equal to 3, we use Oneway-ANOVA method for comparison. When the overall $\mathrm{F}$ test was significant $(P<0.05)$, Tukey's adjustment method was used for post comparison to determine the degree of significant pairwise difference.

\section{Table 2. Antibodies}

\begin{tabular}{lll}
\hline Item & Company & Country \\
\hline p-ULK (ser555), ULK, p-Beclin, Beclin, & Cell Signaling & USA \\
LC3B, AMPK, p-AMPK & Technology & \\
Claudin 3, HO-1, Nrf2 & Abcam & USA \\
Occludin & Thermo Fisher & USA \\
Lamin B, ZO-1, P62 & Proteintech & USA \\
$\beta-T u b u l i n$ & Bosterbio & USA \\
IgG & Beyotime & China \\
$\begin{array}{l}\text { Donkey anti-rabbit IgG }(\mathrm{H}+\mathrm{L}) \\
\text { HRP-conjugated antimouse and } \\
\text { antirabbit secondary antibodies }\end{array}$ & Life Technologies & USA \\
\hline
\end{tabular}

\section{Results}

\section{Binding site of niacin with GPR 109A}

In order to study the biological function of niacin in vivo (S1d). We simulated the binding site between niacin and GPR109A, and found that niacin can activate GPR109A receptor through SER-244 and THR-280, thus playing its biological function (S1a-c).

\section{GPR 109A alleviate pathological injury to the mammary gland}

We performed sections and H\&E staining of mammary tissue (Fig. 1a). In WT and GPR109A-/mice, there was no damage to the mammary glands in the NT and Niacin groups (Fig. 1a). Compared with those from the NT and Niacin group, mammary glands from the LPS group presented with severe pathological injuries, such as thickening of the alveolar wall and neutrophil infiltration (Fig. 1a). Neutrophil infiltration in the LPS group of
GPR109A $\%$ mice was more serious than that in the LPS group of WT mice. Neutrophil infiltration in the LPS + niacin group in WT mice was significantly improved after niacin treatment, but the same effect was not observed in LPS + niacin group in GPR109A $\%$ mice (Fig. 1a). The pathological scores of these mammary glands were also consistent with the results of H\&E staining (Fig. 1b).

\section{GPR 109A decreased the expression of proinflammatory cytokines}

Mammary epithelial cells are opportunistic sentinel cells that play an important role in monitoring the invasion of the mammary gland by pathogenic microorganisms [30]. In WT mice, the levels of MPO, IL-1 $\beta$, IL-6, and TNF- $\alpha$ in mammary gland tissues were higher than other groups (Fig. 2a-f). Compared with the LPS group, niacin treatment remarkably reduced the expression levels of these inflammatory factors (Fig. 2a-h). In GPR109A-/- mice, the MPO, IL-6, IL-1 $\beta$ and TNF- $\alpha$ levels were increased in the LPS and LPS+niacin groups (Fig. 2a-f). The level of TNF- $a$ in GPR109A-/- mice was significantly higher than that in WT mice (Fig. 2c).

\section{GPR 109A repairs the integrity of the blood- milk barrier}

Blood milk barrier is very important in lactating women [31]. In WT mice, FITC-albumin penetrated into the acini in LPS group (Fig. 3a). As expected, niacin could inhibit the LPS-induced barrier damage (Fig. 3a).

In GPR109A-/- mice, there was no fluorescence in acinar lumina in the NT group or Niacin group, but there was a large amount of FITC-albumin in acinar lumina in the LPS group, more than that present in WT mice. After the niacin treatment, we found that there was still a large amount of FITC-albumin in the acinar lumina of GPR109A /- mice (Fig. 3a).
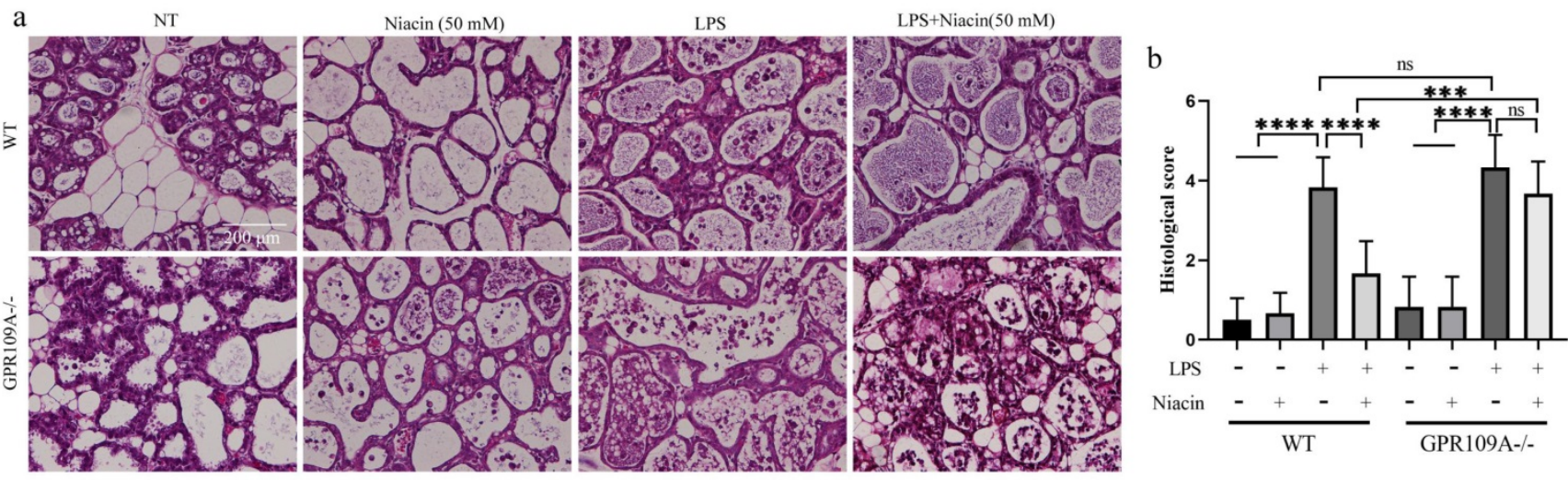

Figure 1. Effects of GPR109A on mice mammary gland injury. (a) The H\&E staining of mammary gland (original magnification $200 \times$ ). (b) The injury score of mammary gland, this is based on the three points previously described.Values are presented as means \pm SD $(* * * p<0.001, * * * * p<0.0001)$. 

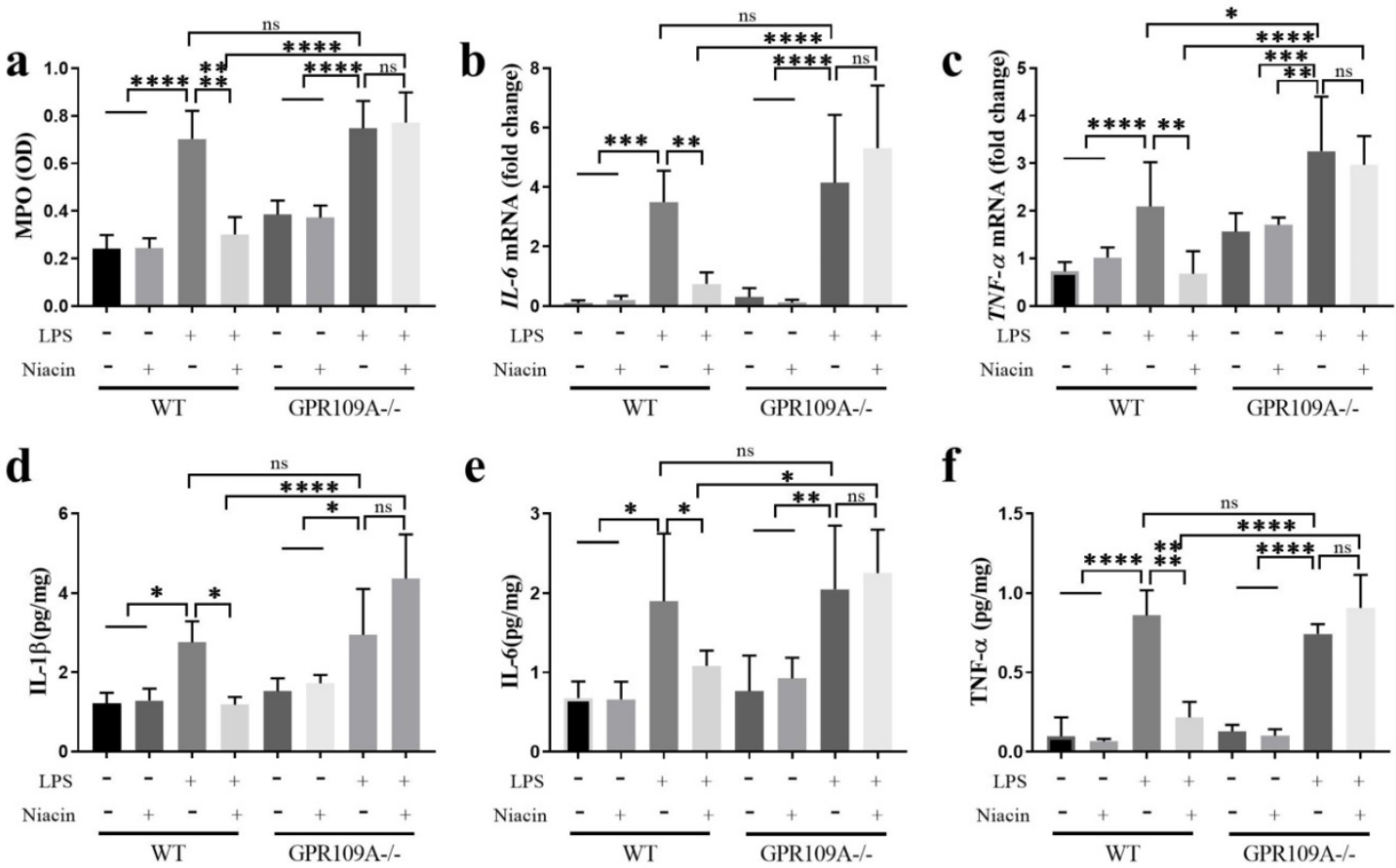

Figure 2. Activating GPR109A can reduce the release of cytokines. (a) Activity of myeloperoxidase (MPO). (b, c) mRNA levels of IL-6 and TNF- $\alpha$. (d-f) The Protein contents of IL-1 $\beta$, TNF- $\alpha$ and IL-6. Values are presented as means \pm SD $(n=5)(* p<0.05, * * p<0.01, * * * p<0.001, * * * * p<0.0001)$.

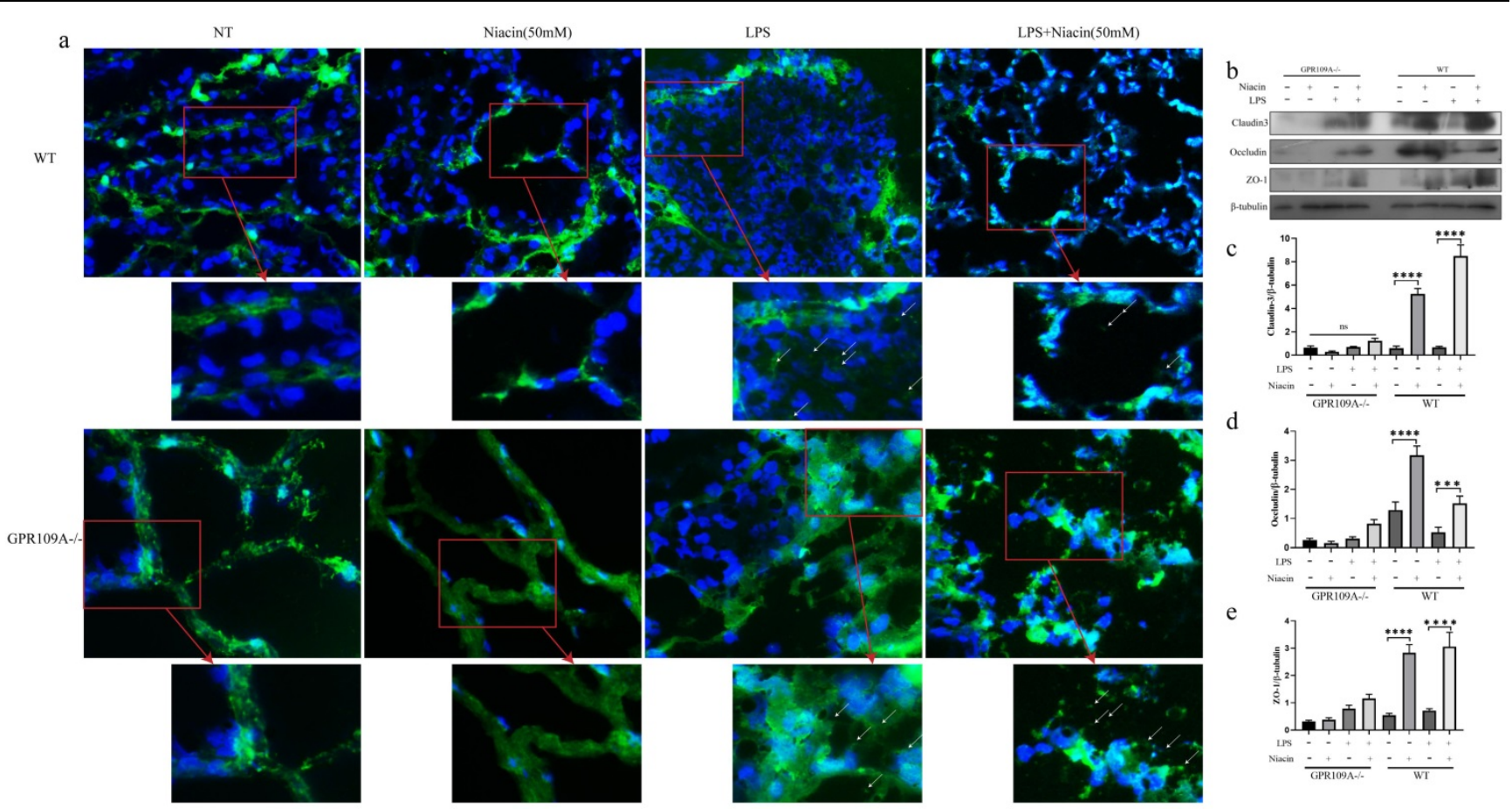

Figure 3. Penetration of FITC-albumin as it enters mammary acini through the blood milk barrier. Mammary glands from the eight groups were treated with previous description. (a) The color green represent FITC-albumin and the colot blue means nuclei (DAPI). After LPS injection, we can observe green FITC-albumin in mammary acini under fluorescence microscope. (b-e) The levels of Occludin, Claudin 3 and ZO-1. Values are presented as means \pm SD (*** $p<0.001$, $* * * * *<0.0001)$.

To further study the effect of GPR109A on the blood milk barrier, we detected the levels of ZO-1, Occludin and Claudin 3. In WT mice, the levels of ZO-1, Occludin, and Claudin 3 in the LPS group were significantly downregulated, while the levels of these proteins was significantly increased after niacin treatment. In GPR109A $\%$ mice, we found that the expression of Claudin 3, ZO-1 and Occludin was very low (Fig. 3b-e). These results suggested that GPR109A may be involved in maintaining the blood milk barrier. 


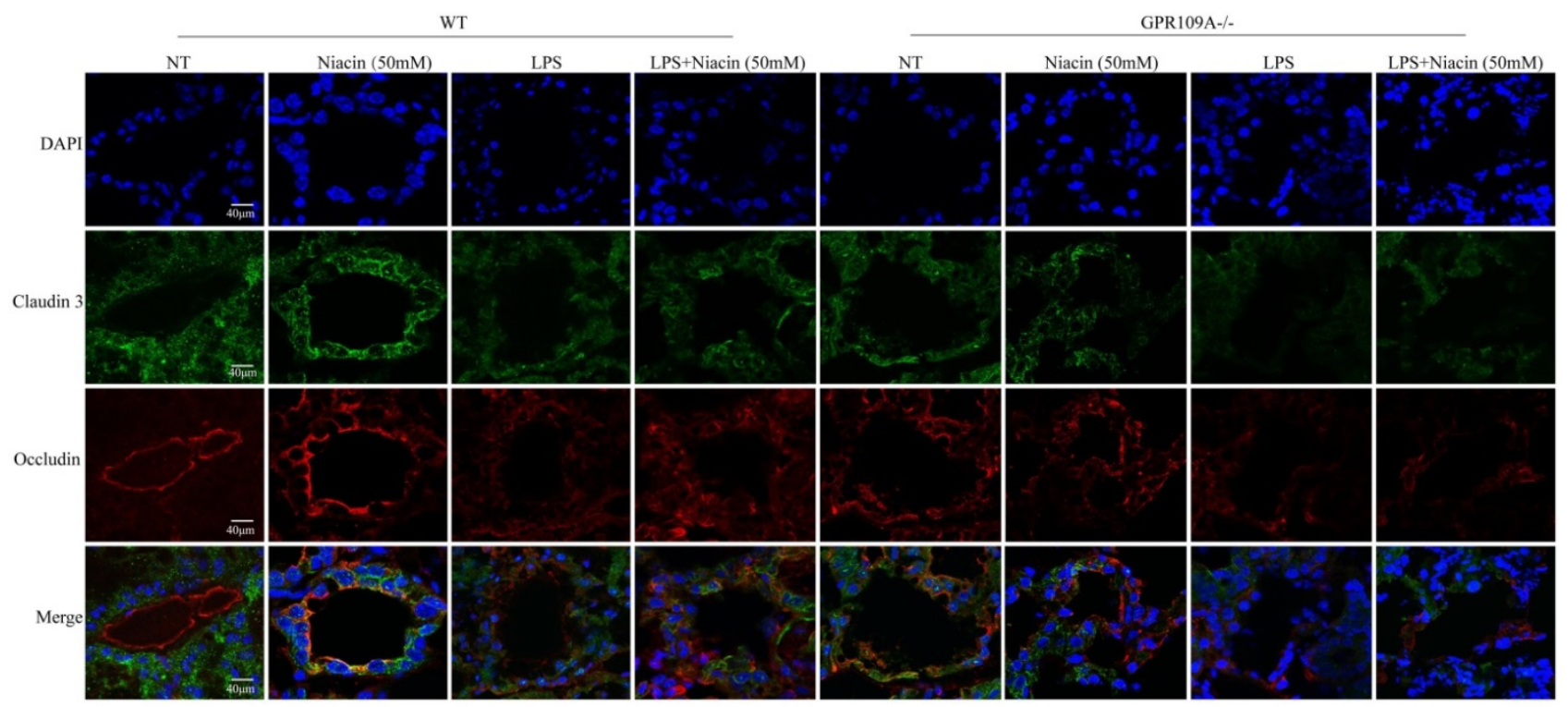

Figure 4. Immunofluorescence results of Claudin 3 and Occludin. Immunofluorescence staining was performed on 8 groups of mammary glands, and the colocalization and fluorescence intensity of Claudin 3 and Occludin in mammary glands were determined.

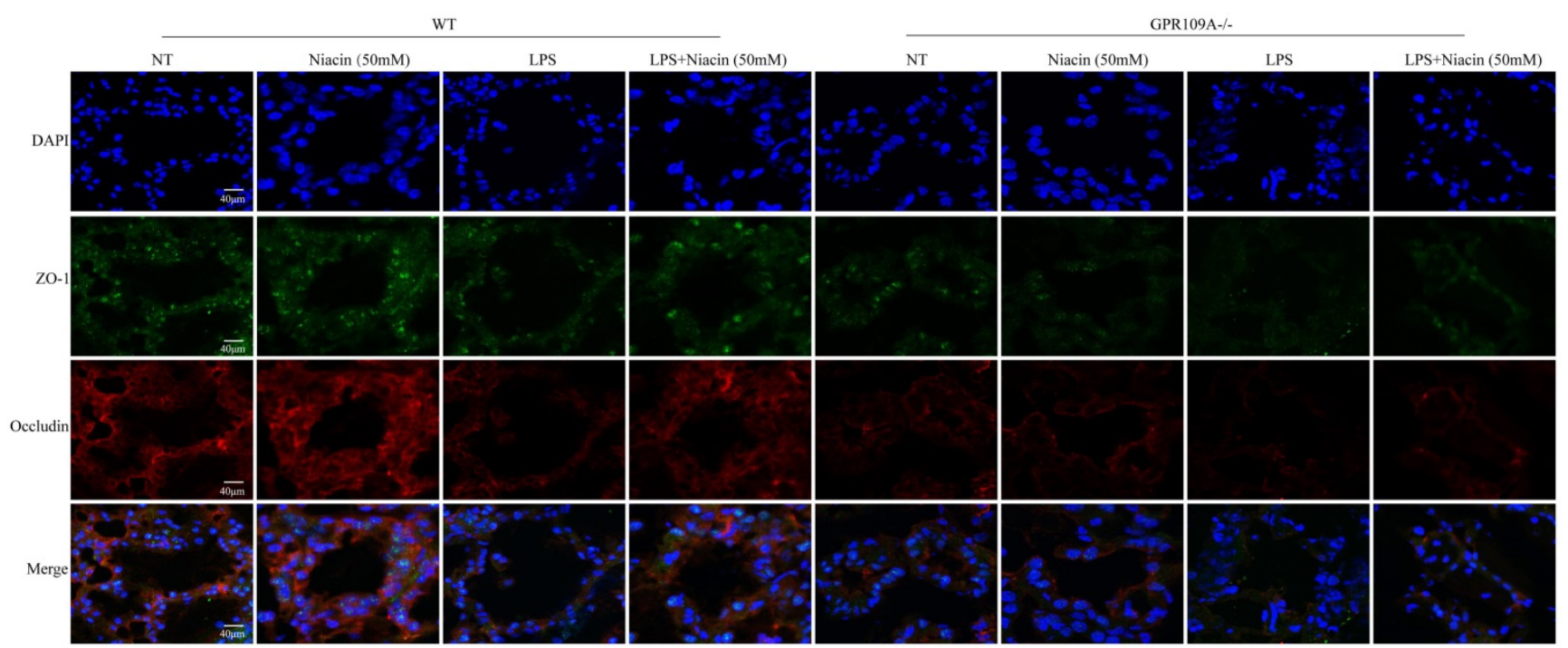

Figure 5. Immunofluorescence results of ZO-1 and Occludin. Immunofluorescence staining was performed on 8 groups of mammary glands, and the colocalization and fluorescence intensity of ZO-1 and Occludin in mammary glands were determined.

Tight junction is an important structure of blood-milk barrier, and they can limit the invasion of microorganisms and regulate the exchange of various substances in the mammary gland. To clarify the mechanism of how GPR109A helps repair the integrity of the blood-milk barrier. In WT mice, the fluorescence intensity of Claudin-3, ZO-1 and Occludin in LPS + niacin group was significantly stronger than that in LPS group, suggesting that niacin repairs tight junction destroyed by LPS (Fig. 4 \& Fig. 5). At the same time, we also found that LPS significantly reduced the fluorescence intensity of ZO-1, occludin and Claudin-3 in mammary gland. Before LPS injection, Claudin-3 and ZO-1 were co-located with occludin in the most-apical region of mammary epithelial cells (MEC) (Fig. 4 \& Fig. 5). We also found that ZO-1 and Claudin-3 in the most-apical region of the junction of MECs could not co-locate with Occludin after injection of LPS 24 hours (Fig. 4 \& Fig. 5). After feeding nacin, the blood milk barrier of LPS injected WT mice was significantly repaired, the expression of ZO-1, Claudin-3 and Occludin was significantly increased, and their localization was partially restored. In GPR $109 \mathrm{~A}^{-/}$mice, the fluorescence intensities of ZO-1, Claudin-3 and Occludin were weaker than those in WT mice (Fig. 4 \& Fig. 5). Moreover, we found that the location of ZO-1, Claudin 3 and Occludin in GPR109A-/- mice's mammary gland were changed (Fig. 4 \& Fig. 5). After LPS injection, niacin could not restore the expression of above proteins in GPR109A $\%$ mice. 

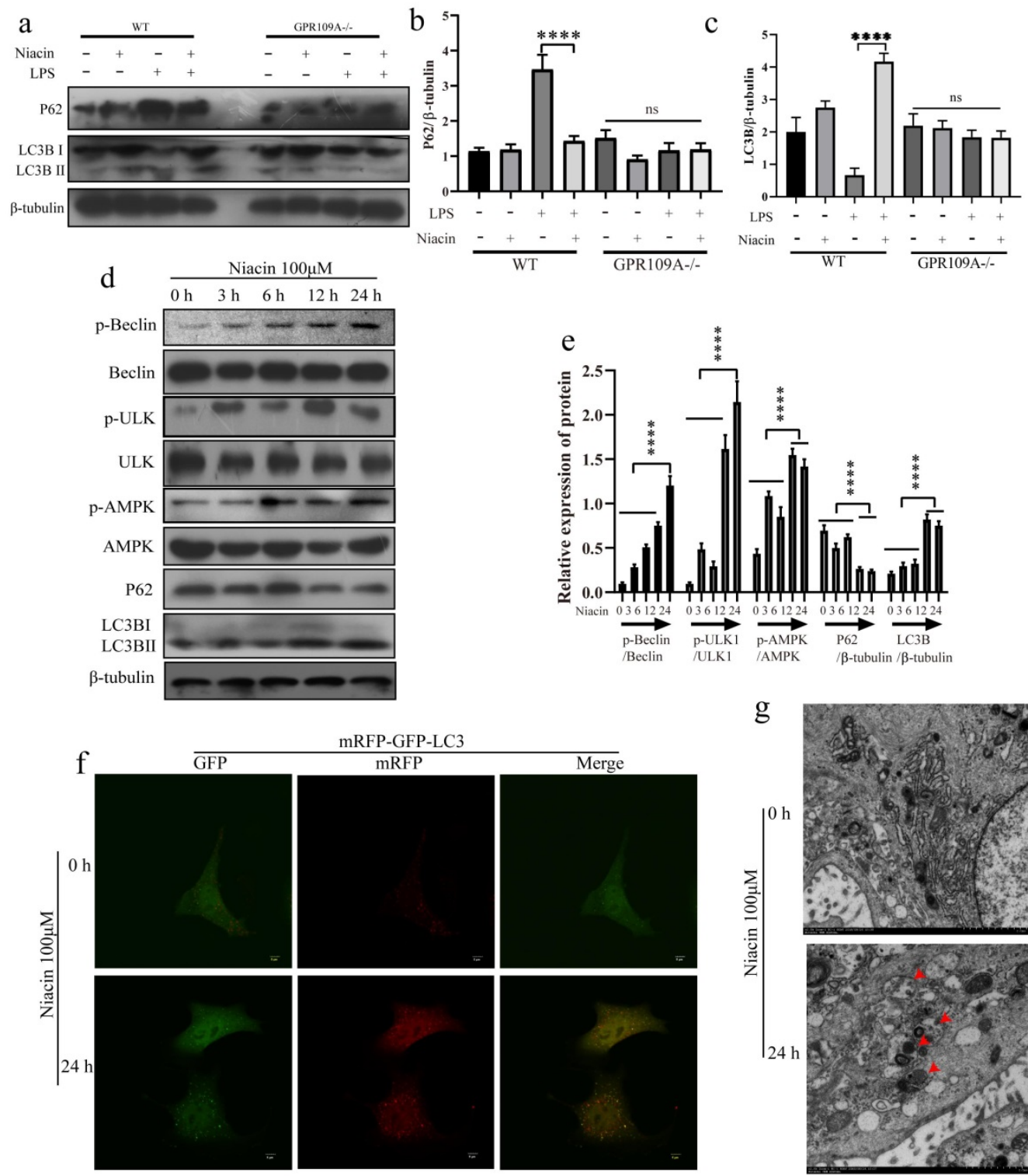

mRFP-GFP-LC3
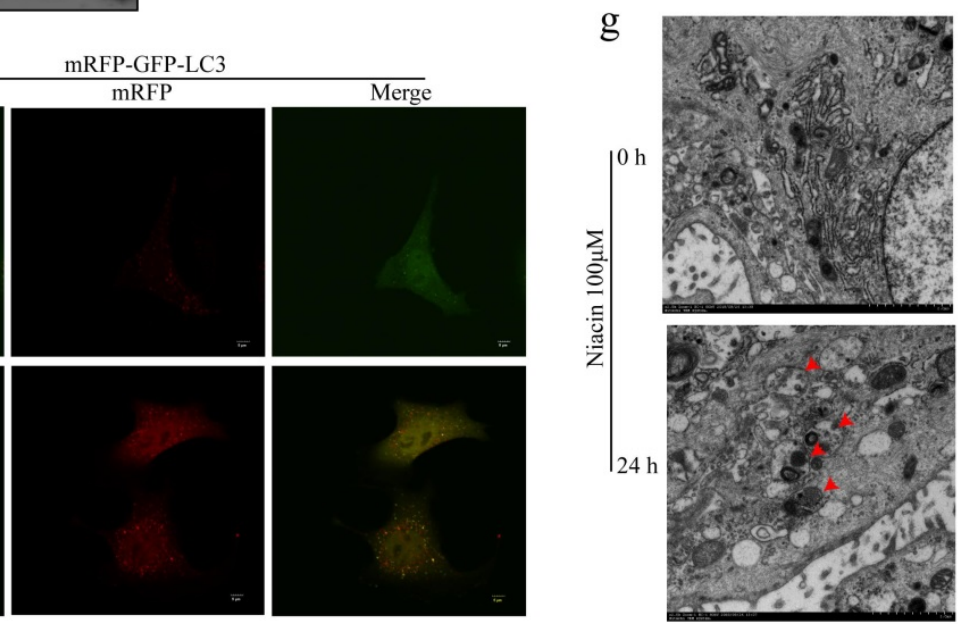

Figure 6. Effect of GPR109A on autophagy. (a-c) Protein levels of p62 and LC3B in the mammary gland. (d, e) Protein levels of p-APMK, AMPK, p-Beclin, Beclin, p-ULK1, ULK 1, P62 and LC3B in niacin treated cells at 0, 3, 6, 12 and 24 hours. (f) After 24 hours of niacin treatment, the autophagy flow of EpH4-Ev increased significantly. (g) Autophagy lysosomes in EpH4-Ev. Values are presented as means $\pm \mathrm{SD}(n=3)(* * * * 0<0.0001)$.

\section{GPR 109A enhances autophagy of mammary epithelial cells}

In vivo, we also found that the protein levels of P62 and LC3B in the mammary glands of GPR109A-/mice significantly decreased, while the protein levels of $\mathrm{P} 62$ and LC3B significantly increased after GPR109A was activated by niacin in WT mice (Fig. 6a-c). To further study the function of GPR109A, we treated EpH4-Ev cells with niacin for 0, 3, 6, 12 or $24 \mathrm{~h}$. Our results showed that niacin could activate autophagy. Niacin enhances autophagy, degradation of P62 and expression of LC3B by promoting the phosphorylation of AMPK, Beclin and ULK1 (Fig. 6d, e). When the mRFP-GFP-lc3 plasmid was transfected into cells, the flow of autophagy was significantly enhanced after treatment with niacin for $24 \mathrm{~h}$ (Fig. 6f). The electron microscopy results also showed that niacin could significantly promote the formation of autophagic lysosomes (Fig. 6g).

\section{GPR 109A activates autophagy through nonclassical pathways}

Studies have shown that autophagy can alleviate inflammation and enhance TJs [32,33]. To further study the anti-inflammatory mechanism of GPR109A, we detected the phosphorylation levels of AMPK, Beclin and ULK1 after knocking down GPR109A. Our results showed that activation of GPR109A promoted phosphorylation of AMPK, Beclin and ULK1 (Fig. 7a, b). The IP test results showed that p-AMPK may be the main factor affecting the phosphorylation of 
Beclin and ULK1 and that the interaction of p-AMPK, p-Beclin and p-ULK1 is also closely related to the activation of GPR109A (Fig. 7c). The results of the IP test were consistent with those of electron microscopy. Activation of GPR109A significantly promoted the formation of autophagic lysosomes (Fig. 7d). To further prove that AMPK is the main downstream effector protein of GPR109A, we inhibited it. After inhibition of AMPK, phosphorylation of AMPK, Beclin and ULK1 was also significantly inhibited (Fig. 7e, f). The autophagy flow results were consistent with the western blotting results (Fig. 7g). These results indicate that GPR109A mainly promotes autophagy through AMPK.

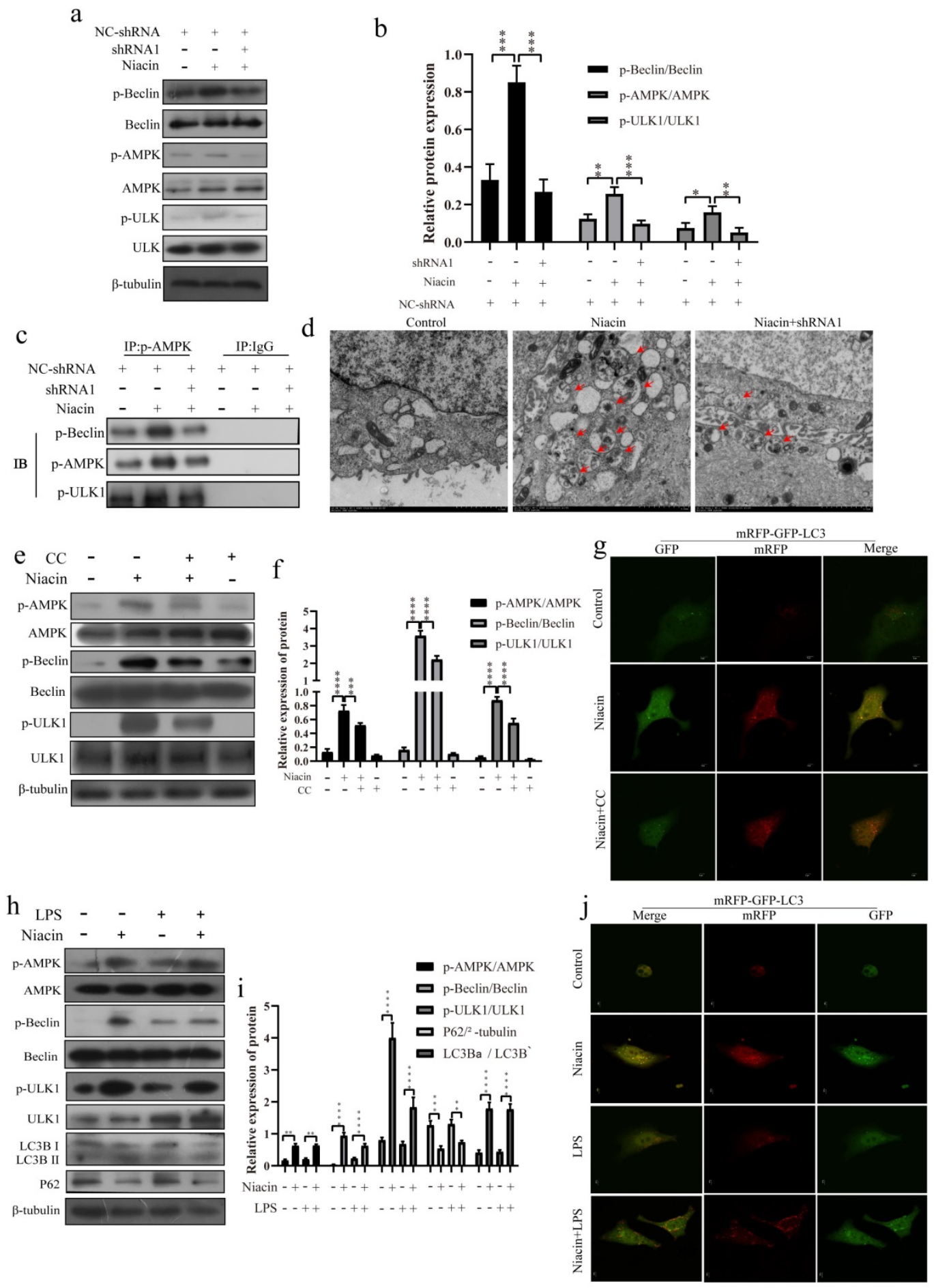

Figure 7. GPR109A inhibits inflammation by activating autophagy through p-AMPK, p-Beclin and p-ULK1. (a, b) The levels of $p-A M P K, p-U L K 1$ and $p-B e c l i n$ after knocking down GPR109A. (c, d) Interaction between p-ULK1, p-Beclin and p-AMPK after knocking down GPR109A. (e-g) Protein levels of p-ULK1 and p-Beclin after inhibition of AMPK. (h) Protein levels of the autophagy related pathway proteins after GPR 109A was activated in LPS-induced MECs. (j) Autophagy flow in the LPS-induced MECs after GPR109A activation. Values are presented as means \pm SD $(n=3)(* p<0.05, * * p<0.01, * * * p<0.001, * * * * p<0.0001)$. 

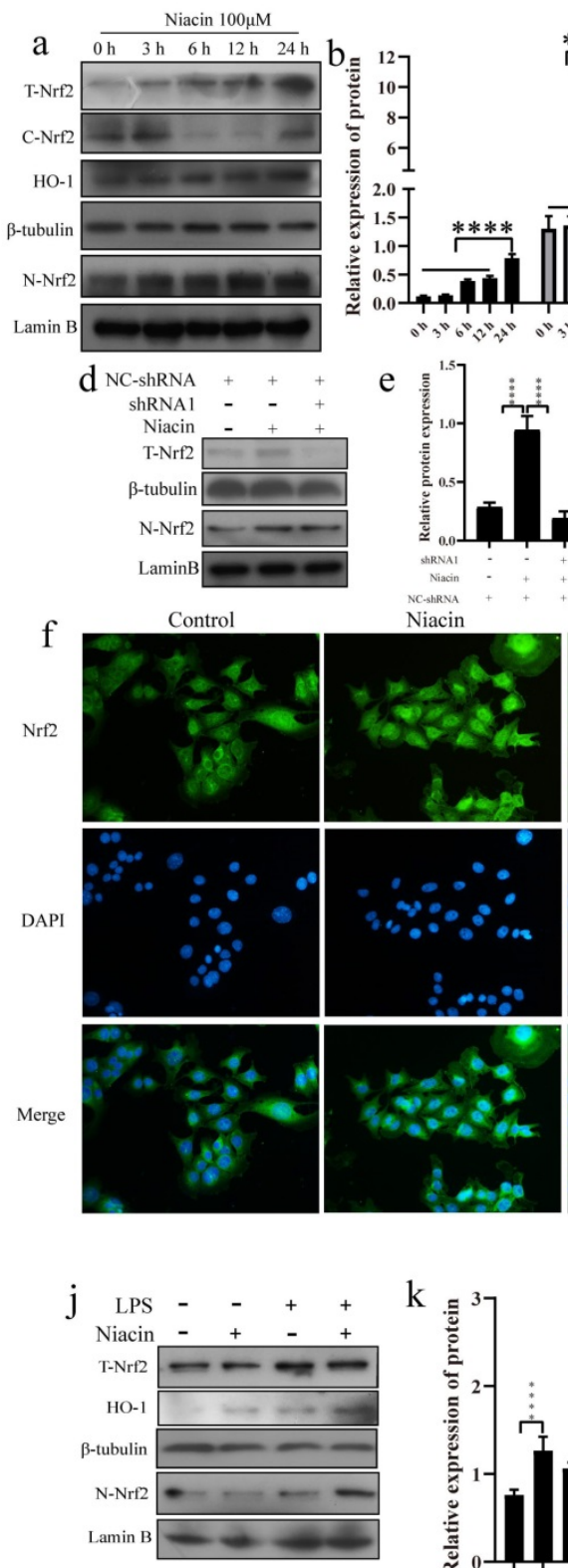

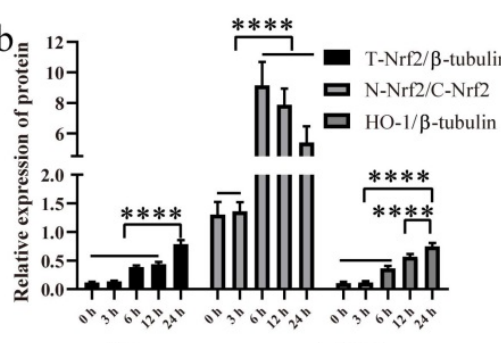

e

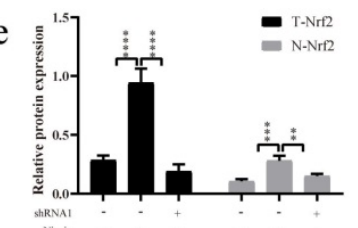

Niacin+shRNA1

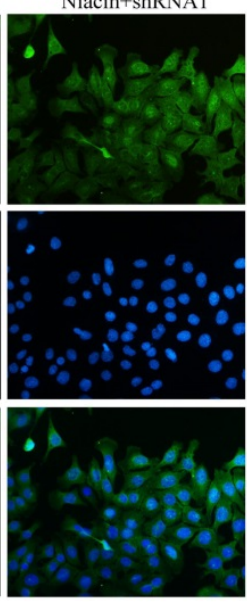

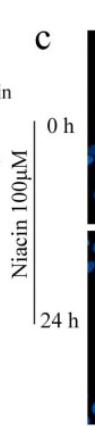
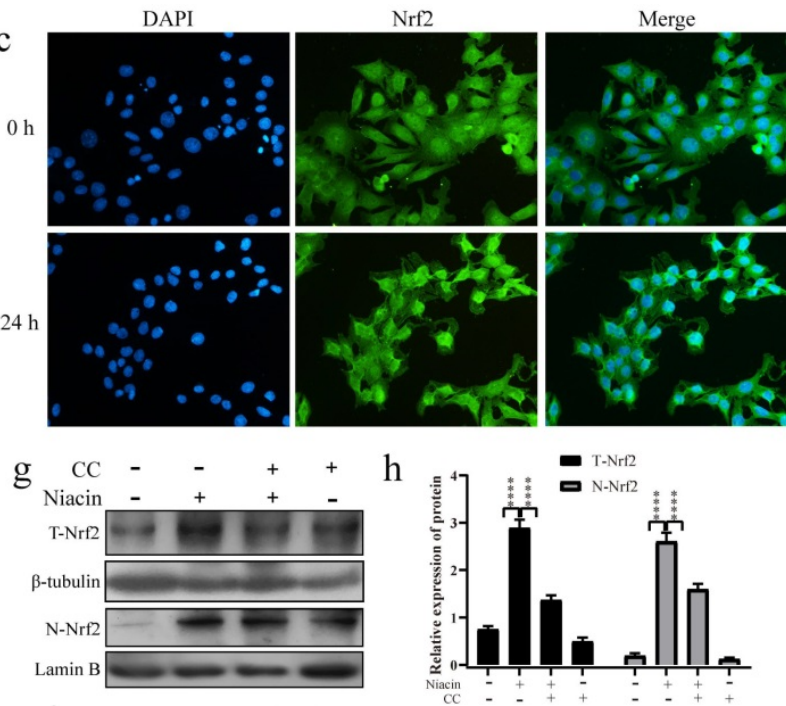

i
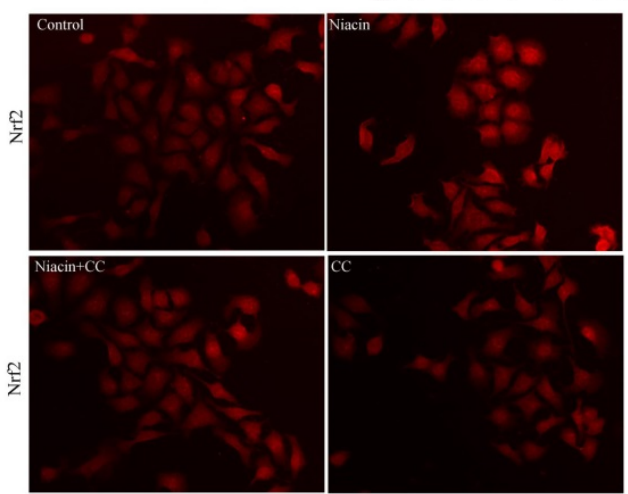

1
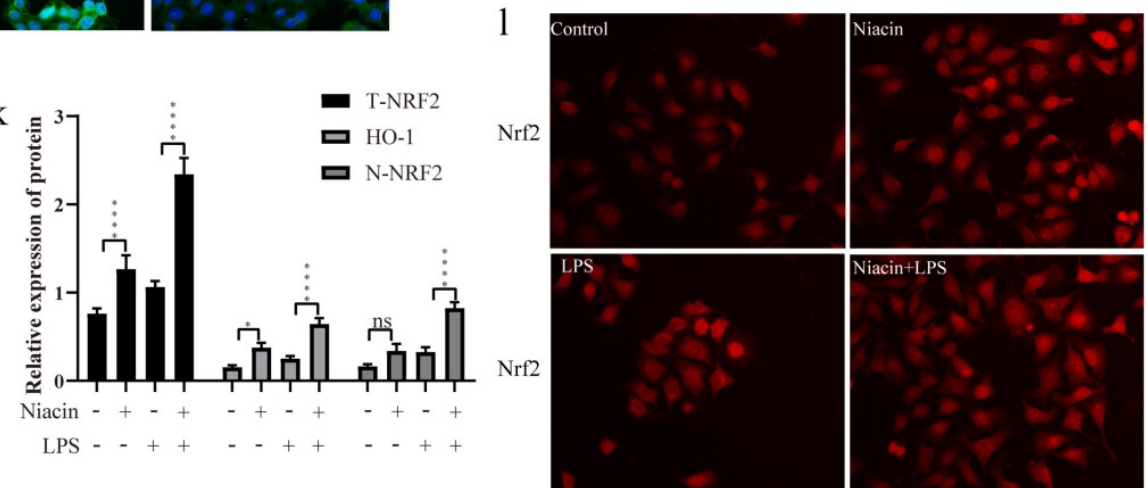

Figure 8. GPR109A promotes Nrf2 nuclear import to exert anti-inflammatory effect by phosphorylating AMPK. (a, b) Protein levels of T-Nrf2, C-Nrf2, HO-1 and N-Nrf2. (c) Immunofluorescence results of Nrf2 nuclear import after treating with niacin for 24 hours. (d, e) The levels of N-Nrf2 and T-Nrf2. (f) Immunofluorescence results of Nrf2 nuclear import after knocking down GPR109A. $(\mathbf{g}, \mathbf{h})$ The levels of N-Nrf2 and T-Nrf2. (i) Immunofluorescence results of Nrf2 nuclear import after treating with niacin, CC or niacin+CC for 24 hours. (j, k) Protein levels of T-Nrf2, HO-1 and N-Nrf2. (I) Immunofluorescence results of Nrf2 nuclear import after treating with LPS, niacin or niacin+LPS for 24 hours. Values are presented as means \pm SD $(n=3)(* p<0.05, * * p<0.01, * * * p<0.001, * * * * 00.0001)$.

To further study the mechanism of GPR109A in alleviating inflammation, we constructed an LPS induced inflammatory response model of EpH4-Ev. After activating GPR109A, the mechanism of GPR109A in the inflammatory response model was studied. We found that activation of GPR109A significantly promoted the phosphorylation of autophagy related proteins (AMPK, Beclin and ULK1) and the degradation of p62 and transformation of LC3BI into LC3BII (Fig. 7h, i). After transfection of the mRFP-GFP-LC3 plasmid, we found that GPR109A activation significantly enhanced autophagy (Fig. 7j).

\section{GPR109A can activate the Nrf2 signaling pathway}

We also found that GPR109A can promote Nrf2 nuclear import. To further study the function of GPR109A, we treated EpH4-Ev cells with niacin for 0, $3,6,12$ or $24 \mathrm{~h}$. Our results showed that niacin could promote the expression of T-Nrf2 (Total-Nrf2), N-Nrf2 (Nuclear-Nrf2) and HO-1 (Fig. 8a, b). After 24 $\mathrm{h}$, the fluorescence intensity in the nucleus was 
significantly higher than that at $0 \mathrm{~h}$ (Fig. 8c). After GPR109A knockdown, the expression of T-Nrf2 and N-Nrf2 significantly decreased (Fig. 8d, e). The immunofluorescence results also showed that Nrf2 nuclear import was significantly inhibited after GPR109A was knocked down (Fig. 8f). Interestingly, T-Nrf2 and N-Nrf2 expression also significantly decreased after AMPK inhibition (Fig. 8g, h). The immunofluorescence results were consistent with the western blotting results (Fig. 8i), suggesting that GPR109A might regulate the nuclear import of Nrf2 through the AMPK signaling pathway. We also verified this hypothesis in the model of the inflammatory response of mammary epithelial cells constructed by treatment with LPS. The immunofluorescence and western blot results showed that the LPS + niacin group had significantly enhanced Nrf2 nuclear import and increased expression of HO-1, thus playing an anti-inflammatory role (Fig. 8j-1).

\section{GPR 109A plays an anti-inflammatory role through Nrf2 and autophagy}

The release of proinflammatory mediators could aggravate inflammation [34]. To further explore the important function of GPR109A in anti-inflammation, we detected proinflammatory mediators in the Eph4-Ev cell line. The results showed that niacin significantly reduced the mRNA expression levels of TNF- $\alpha, I L-1 \beta, I L-6, C O X-2$ and iNOS after $24 \mathrm{~h}$ of niacin treatment, and the anti-inflammatory effect of niacin was significantly weakened after GPR109A was knocked down. These results indicated that GPR109A plays an important anti-inflammatory role after activation (Fig. 9a-e). Moreover, we found that the expression of $I L-1 \beta, I L-6, T N F-\alpha$ and COX-2 was significantly increased after AMPK inhibition, but not
iNOS (Fig. 9f-j). However, the expression of these inflammatory genes increased slightly when autophagy and Nrf2 were inhibited (Fig. 9f-j). These results suggest that GPR109A alleviates inflammation through the AMPK/Nrf2 and AMPK/autophagy signaling pathways.

\section{GPR 109A enhances the tight junction (TJ) of mammary epithelial cells}

To explore the effect of GPR109A on TJs, we detected the protein expression and fluorescence intensity of Occludin, ZO-1 and Claudin 3. The results showed that the expression of Occludin, Claudin 3 and ZO-1 were significantly increased after niacin treatment (Fig. 10a, b). The fluorescence intensities of Occludin, ZO-1 and Claudin-3 significantly increased after niacin treatment for $24 \mathrm{~h}$ (Fig. 10c). These results indicate that the levels of Occludin, Claudin-3 and ZO-1 in mammary epithelial cells significantly increased after GPR109A was activated and significantly decreased after GPR109A was knocked down (Fig. 10d, e). The fluorescence intensity of these proteins was consistent with the western blotting results (Fig. 10g). The transmission electron microscope (TEM) results also showed that the TJs were strengthened after GPR109A was activated (Fig. 10f).

To further understand how GPR109A enhances TJs, we added inhibitors of autophagy (3-MA, $5 \mu \mathrm{M})$, AMPK (CC, $5 \mu \mathrm{M})$ and Nrf2 (ML385, $5 \mu \mathrm{M}$ ). The results showed that inhibition of AMPK and Nrf2 significantly reduced the protein levels of the TJs, indicating that the AMPK signaling pathway and autophagy play important roles in TJs (Fig. 10h, i). Nrf2 could regulate the expression of Occludin (Fig. $10 \mathrm{~h}, \mathrm{i})$.
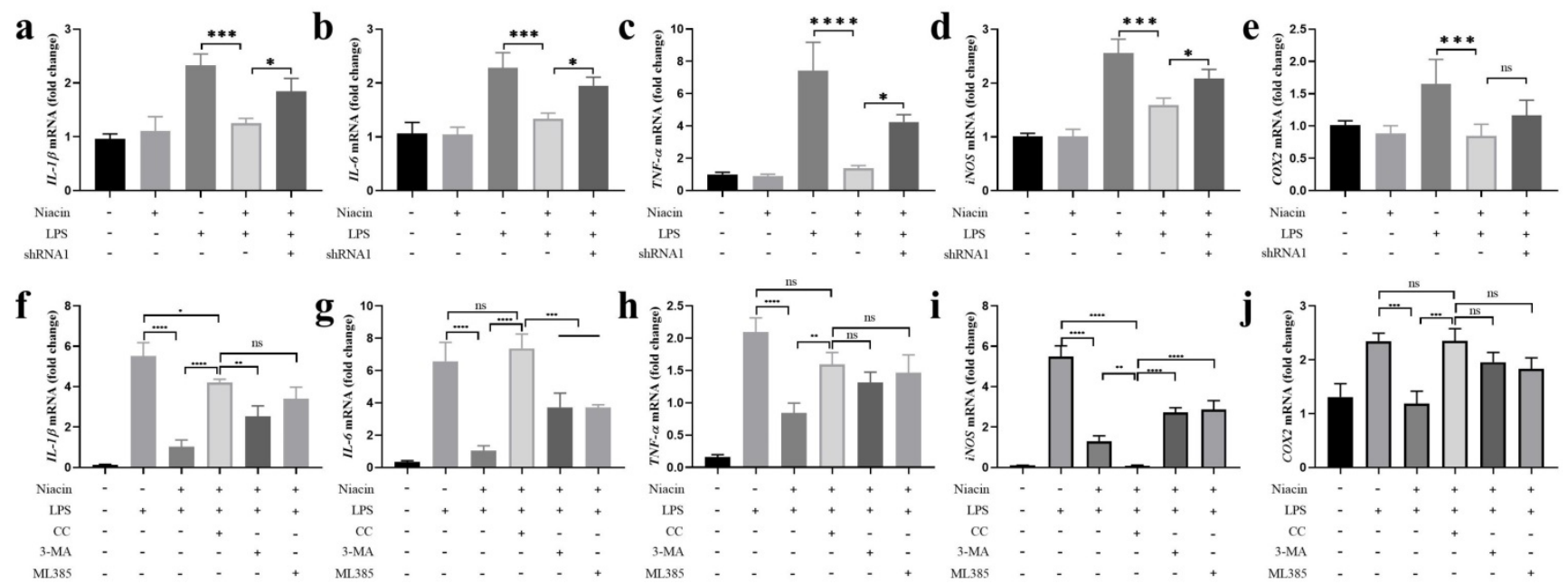

Figure 9. GPR 109A could exert anti-inflammatory effect by activating autophagy and Nrf2 nuclear import through AMPK signal pathway. (a-e) The levels of TNF- $\alpha, I L-I \beta, I L-6, C O X-2$ and iNOS were detected using qRT-PCR in EpH-Ev ( $n=3)$. (f-j) Gene levels of TNF- $\alpha$, iNOS, COX-2, IL-6 and IL-I $\beta$ were detected using qRT-PCR in $\mathrm{EpH}-\mathrm{Ev}(\mathrm{n}=3)$. Values are presented as means $\pm \mathrm{SD}\left(*_{p}<0.05, * * p<0.01, * * * p<0.001, * * * * p<0.0001\right)$. 

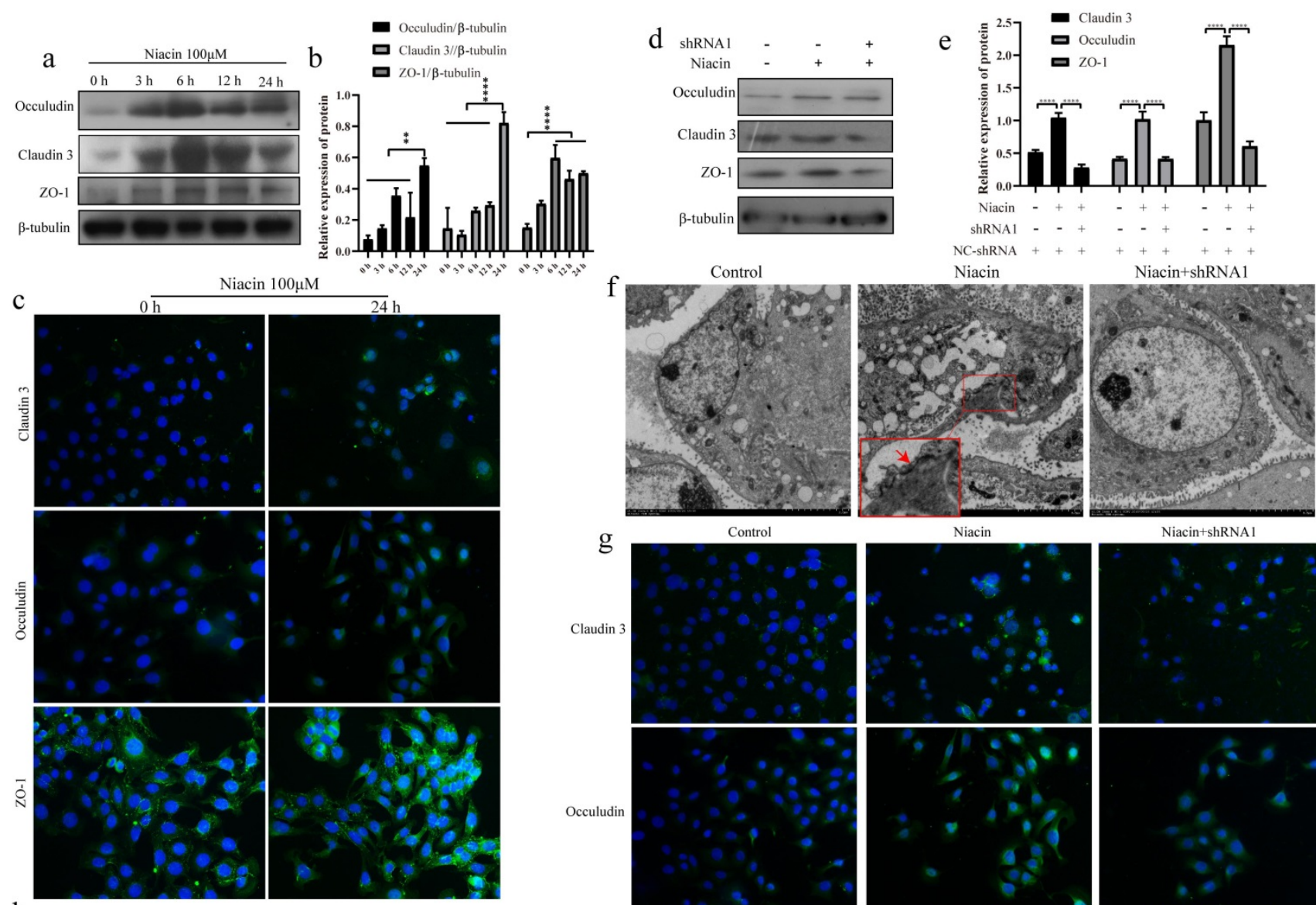

h
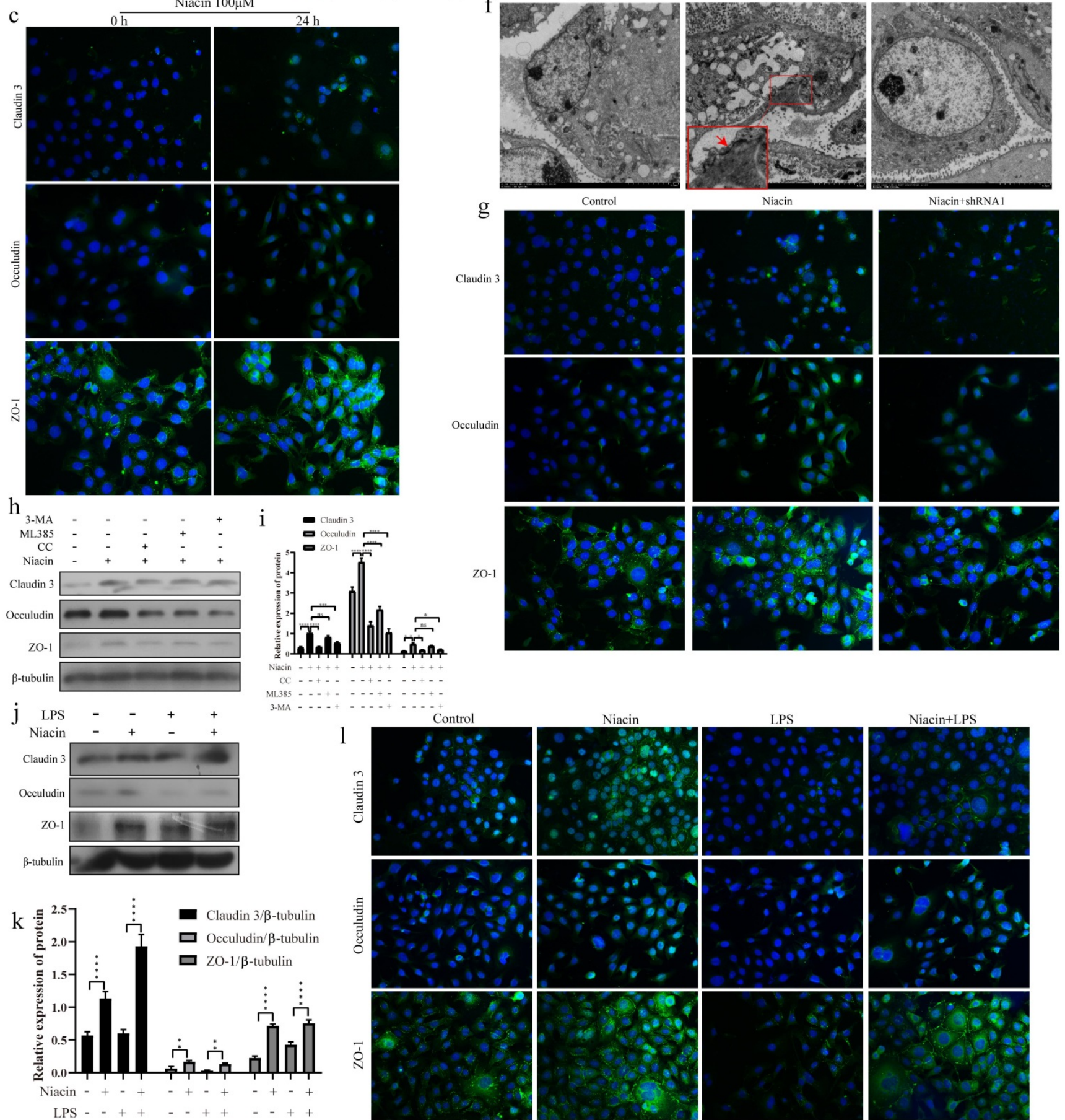

Figure 10. GPR109A enhances tight junction protein expression by activating autophagy and Nrf2. (a, b) The levels of ZO-1, Claudin 3 and Occludin after niacin treatment for 0, 3, 6, 12 and 24 hours. (c) Immunofluorescence results of Claudin 3, ZO-1 and Occludin after niacin treatment for 24 hours. (d, e) The levels of ZO-1, Claudin 3 and Occludin after knocking down GPR 109A. (f) The results of electron microscopy show that the tight junction was strengthened after adding niacin. (g) Immunofluorescence results of Claudin 3, ZO-1 and Occludin after knocking down GPR 109A. (h, i) The levels of Occludin, Claudin 3 and ZO-1 in EpH-Ev cells that were treated with niacin, niacin+3-MA, niacin +ML385 or niacin+CC. (j, k) The levels of Occludin, Claudin 3 and ZO-1 after the cells were treated with LPS, niacin or niacin+LPS. Values are presented as means $\pm \mathrm{SD}(\mathrm{n}=3)(* p<0.05, * * p<0.01, * * * p<0.001, * * * * p<0.0001)$. 
In MECs, we also found that GPR109A could protect the integrity of the blood milk barrier and promote the expression of TJs. The results showed that the levels of Claudin 3, ZO-1 and Occludin in the LPS + niacin group significantly increased (Fig. 10j, k). The immunofluorescence results also showed that GPR109A could promote the expression of TJs (Fig. 101) and that this function was mainly mediated through the AMPK/autophagy signaling pathway.

\section{Discussion}

In this study, we found that the integrity of the blood milk barrier and the level of autophagy of the GPR109A $\%$ mice's mammary gland significantly decreased and that the inflammatory response of the GPR109A $\%$ mice significantly increased after LPS stimulation. These results showed that GPR109A plays important roles in improving the blood milk barrier, in alleviating inflammation and in changing autophagy. Our other experiments showed that GPR109A could regulate the phosphorylation of ULK1, Beclin and AMPK to improve mastitis and enhance the blood milk barrier. Moreover, GPR109A could also promote the expression of Nrf2 and HO-1, thus playing an anti-inflammatory role. Therefore, the results of our study suggested that GPR109A might alleviate inflammation and enhance the blood milk barrier by activating autophagy and promoting Nrf2 nuclear import.

Autophagy is an important means by which cells cope with the internal and external environments [35]. When cells sense changes in their internal and external environment, they can maintain homeostasis and the healthy operation of organelles by regulating their autophagy level 36 . When mammary epithelial cells are stimulated by LPS, they produce a strong inflammatory response and release a large number of proinflammatory mediators [37]. These proinflammatory mediators may change the microenvironment inside and outside cells [38], destroy the blood milk barrier, and intensify the inflammatory response [39]. Some studies have shown that autophagy can maintain the healthy operation of cells [40], enhance the blood milk barrier and reduce the inflammatory response by clearing aging and damaged organelles [41,42]. It has been shown that activation of GPR109A can reduce inflammation and improve the symptoms of ulcerative colitis [15]. Although the anti-inflammatory function of GPR109A has been reported, the specific mechanism of how GPR109A acts as an anti-inflammatory agent and enhances the blood milk barrier is not clear. Until this study, there were no available reports showing that GPR109A plays a role in autophagy, the blood milk and barrier mastitis.
To further explore the biological function of GPR109A, we found that GPR109A activation could significantly enhance the blood milk barrier and alleviate mastitis in WT mice. The level of autophagy and the integrity of the blood milk barrier in GPR109A $\%$ mice were both significantly inhibited, while the level of inflammation was significantly increased, indicating that the anti-inflammatory function of GPR109A in the mammary gland might be closely related to autophagy. We also found that niacin significantly inhibited mastitis and enhanced the blood milk barrier, but these effects disappeared after GPR109A was knocked out, suggesting that niacin could alleviate inflammation and enhance the blood milk barrier by activating GPR109A. This result supported the hypothesis that GPR109A could reduce inflammation and protect blood milk barrier.

Some studies have shown that both autophagy and Nrf2 can alleviate inflammation [43]. Autophagy can alleviate the inflammatory response by removing necrotic or aged organelles and foreign bodies from cells. Autophagy can be divided into noncanonical and canonical pathways [44]. In our study, GPR109A was found to significantly promote the phosphorylation of AMPK, so we detected the phosphorylation of Beclin and ULK. The results showed that GPR109A could activate p-Beclin and p-ULK through p-AMPK. We also observed an increase in the flow of autophagy after applying niacin. However, the flow of autophagy significantly decreased after GPR109A was knocked down. Although previous studies have shown that autophagy can alleviate inflammation [45], there is no available study on GPR109A and autophagy.

We found that GPR109A could alleviate mastitis and enhance the blood milk barrier by activating autophagy. Nrf2 is a very important antioxidant related transcription factor. Nrf2 can alleviate inflammation by promoting the expression of its downstream anti-inflammatory genes [46]. The Nrf2/ HO-1 signaling could significantly reduce various inflammation according to many studies [47]. Lv et al. found that isoliquiritigenin can promote the phosphorylation of AMPK and Nrf2 nuclear import in RAW264.7 cells to alleviate lung injury [48]. Our study found that GPR109A could also increase the expression of HO-1 through phosphorylation of AMPK in Eph4-Ev. Our experiments showed that GPR109A can exert its anti-inflammatory function through autophagy and Nrf2 and that this function is mainly realized by activating the AMPK signaling pathway.

The blood milk barrier, which plays an important role during lactation, is very important in mother and baby. Some studies have shown that 


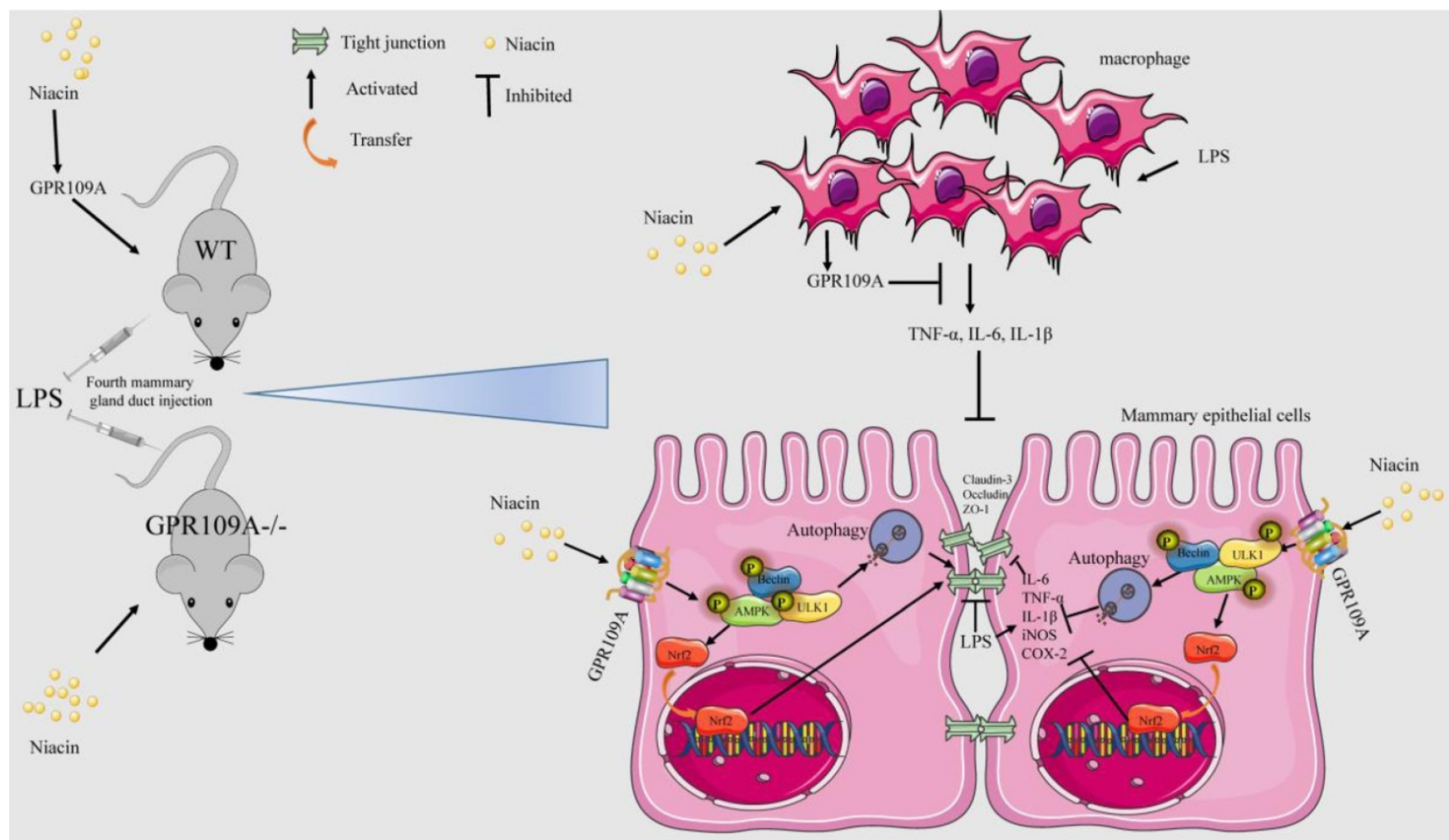

Figure 11. The mechanism of niacin in anti-mastitis and enhanced blood milk barrier. GPR109A can alleviate mastitis mainly through two ways. On the one hand, it can alleviate the inflammatory response of mammary epithelial cells and protect the integrity of blood milk barrier by activating AMPK/Nrf2 and autophagy. On the other hand, it can reduce the damage of mammary epithelial cells by inhibiting the release of pro-inflammatory mediators by macrophages.

autophagy can enhance the tight junction of the intestinal epithelium [49]. Other studies have shown that a lack of Nrf2 can seriously damage the esophagus of mice [50]. These studies indicate that Nrf2 also participates in the repair of the barrier. Our research also found that GPR109A can enhance the barrier function through autophagy and Nrf2. Interestingly, we found that the protection of the blood milk barrier by autophagy is better than that mediated by Nrf2. Since niacin promotes autophagy and Nrf2 nuclear import, autophagy and Nrf2 play a synergistic role in enhancing the blood milk barrier.

To further clarify the anti-inflammatory function of GPR109A, we found that different concentrations of niacin could significantly reduce the expression of pro-inflammatory cytokines in RAW264.7 cells. Interestingly, we found that the supernatant of LPS treated RAW264.7 cells could also promote the inflammatory response in mammary epithelial cells, while GPR109A could significantly inhibit the expression of pro-inflammatory cytokines caused by the supernatant (S 3-S 5).

In conclusion, our results showed that niacin can target GPR109A to alleviate mastitis and enhance the blood milk barrier through AMPK/Nrf2 and AMPK/ autophagy. Moreover, GPR109A can significantly reduce the damage to mammary epithelial cells caused by the macrophage supernatant (Fig. 11).

\section{Supplementary Material}

Supplementary figures.

http://www.ijbs.com/v17p4271s1.pdf

\section{Acknowledgements}

\section{Funding}

National Natural Science Foundation of China, Grant/Award Numbers: 31873004, 32172807, 32102624; Jilin Scientific and Technological Development Program, 20200201111JC; Postdoctoral Research Foundation of China (CN), Grant/Award Numbers: 2020M681043, 2020M681044. Jilin Young Scientific and Technological Talents Promotion Project: QT202127.

\section{Author Contributions}

Wenjin Guo and Shoupeng $\mathrm{Fu}$ designed experiments.Wenjin Guo, Xingchi Kan, Yingchun Su, Yu Cao, Xin Ran, Shu Liu and Wen Li carried out experiments. Wenjin Guo analyzed experimental results. Wenjin Guo analyzed sequencing data. Wenjin Guo, Shoupeng Fu and Juxiong Liu wrote the manuscript.

\section{Competing Interests}

The authors have declared that no competing interest exists. 


\section{References}

1. Spencer JP. Management of mastitis in breastfeeding women. Am Fam Physician. 2008;78(6):727-731.

2. Beltran Vaquero DA, Crespo Garzon AE, Rodriguez Bravo TC, Garcia Iglesias A. [Infectious mastitis: a new solution for an old problem]. Nutricion hospitalaria. 2015;31 Suppl 1:89-95.

3. Laas E, Touboul C, Kerdraon O, Catteau-Jonard S. [Inflammatory and infectious breast mastitis outside of pregnancy and lactation: Guidelines]. Journal de gynecologie, obstetrique et biologie de la reproduction. 2015;44(10):996-1016.

4. Tena D, Lopez-Garrido B, Losa C. Clinical mastitis in breastfeeding women: study of 56 cases. Infect Dis (Lond). 2016;48(11-12):867-868.

5. Berens PD. Breast Pain: Engorgement, Nipple Pain, and Mastitis. Clinical obstetrics and gynecology. 2015;58(4):902-914.

6. Child WHODo, Health A, Development. Mastitis : causes and management. 2000.

7. Tsugami Y, Matsunaga K, Suzuki T, Nishimura T, Kobayashi K. Phytoestrogens Weaken the Blood-Milk Barrier in Lactating Mammary Epithelial Cells by Affecting Tight Junctions and Cell Viability. J Agric Food Chem. 2017;65(50):11118-11124.

8. Zhang C, Zhai S, Wu L, et al. Induction of size-dependent breakdown of blood-milk barrier in lactating mice by TiO2 nanoparticles. PLoS One. 2015;10(4):e0122591.

9. Kobayashi K, Oyama S, Numata A, Rahman MM, Kumura H. Lipopolysaccharide disrupts the milk-blood barrier by modulating claudins in mammary alveolar tight junctions. PLoS One. 2013;8(4):e62187.

10. Wall SK, Hernandez-Castellano LE, Ahmadpour A, Bruckmaier RM, Wellnitz O. Differential glucocorticoid-induced closure of the blood-milk barrier during lipopolysaccharide- and lipoteichoic acid-induced mastitis in dairy cows. J Dairy Sci. 2016;99(9):7544-7553.

11. Wang JJ, Wei ZK, Zhang X, Wang YN, Fu YH, Yang ZT. Butyrate protects against disruption of the blood-milk barrier and moderates inflammatory responses in a model of mastitis induced by lipopolysaccharide. British journal of pharmacology. 2017;174(21):3811-3822.

12. Wu BJ, Chen K, Barter PJ, Rye KA. Niacin inhibits vascular inflammation via the induction of heme oxygenase-1. Circulation. 2012;125(1):150-158.

13. Jia D, Bai $\mathrm{P}$, Wan $\mathrm{N}$, et al. Niacin Attenuates Pulmonary Hypertension Through H-PGDS in Macrophages. Circ Res. 2020;127(10):1323-1336.

14. Bahouth SW, Nooh MM. Barcoding of GPCR trafficking and signaling through the various trafficking roadmaps by compartmentalized signaling networks. Cell Signal. 2017;36:42-55

15. Chen G, Ran X, Li B, et al. Sodium Butyrate Inhibits Inflammation and Maintains Epithelium Barrier Integrity in a TNBS-induced Inflammatory Bowel Disease Mice Model. EBioMedicine. 2018;30:317-325.

16. Fu SP, Wang JF, Xue WJ, et al. Anti-inflammatory effects of BHBA in both in vivo and in vitro Parkinson's disease models are mediated by GPR109A-dependent mechanisms. I Neuroinflammation. 2015;12:9.

17. Elangovan S, Pathania R, Ramachandran S, et al. The niacin/butyrate receptor GPR109A suppresses mammary tumorigenesis by inhibiting cell survival. Cancer Res. 2014;74(4):1166-1178.

18. Murakami S, Motohashi H. Roles of Nrf2 in cell proliferation and differentiation. Free Radic Biol Med. 2015;88(Pt B):168-178.

19. Tonelli C, Chio IIC, Tuveson DA. Transcriptional Regulation by Nrf2. Antioxid Redox Signal. 2018;29(17):1727-1745.

20. Kerins MJ, Ooi A. The Roles of NRF2 in Modulating Cellular Iron Homeostasis. Antioxid Redox Signal. 2018;29(17):1756-1773.

21. Buendia I, Michalska P, Navarro E, Gameiro I, Egea J, Leon R. Nrf2-ARE pathway: An emerging target against oxidative stress and neuroinflammation in neurodegenerative diseases. Pharmacology \& therapeutics. 2016;157:84-104.

22. Li SQ, Feng L, Jiang WD, et al. Deficiency of dietary niacin impaired gill immunity and antioxidant capacity, and changes its tight junction proteins via regulating NF-kappaB, TOR, Nrf2 and MLCK signaling pathways in young grass carp (Ctenopharyngodon idella). Fish Shellfish Immunol. 2016;55:212-222.

23. Parzych KR, Klionsky DJ. An overview of autophagy: morphology, mechanism, and regulation. Antioxid Redox Signal. 2014;20(3):460-473.

24. Levine B, Mizushima N, Virgin HW. Autophagy in immunity and inflammation. Nature. 2011:469(7330):323-335.

25. Liu F, Nie C, Zhao N, et al. MiR-155 Alleviates Septic Lung Injury by Inducing Autophagy Via Inhibition of Transforming Growth Factor-beta-Activated Binding Protein 2. Shock (Augusta, Ga). 2017;48(1):61-68.

26. Chan Y, Chen W, Wan W, Chen Y, Li Y, Zhang C. Abeta1-42 oligomer induces alteration of tight junction scaffold proteins via RAGE-mediated autophagy in bEnd.3 cells. Experimental cell research. 2018;369(2):266-274.

27. Guo W, Liu B, Yin Y, et al. Licochalcone A Protects the Blood-Milk Barrier Integrity and Relieves the Inflammatory Response in LPS-Induced Mastitis. Front Immunol. 2019;10:287.

28. Guo W, Liu B, Hu G, et al. Vanillin protects the blood-milk barrier and inhibits the inflammatory response in LPS-induced mastitis in mice. Toxicol Appl Pharmacol. 2019;365:9-18.

29. Guo W, Liu J, Li W, et al. Niacin Alleviates Dairy Cow Mastitis by Regulating the GPR109A/AMPK/NRF2 Signaling Pathway. Int J Mol Sci. 2020;21(9).

30. Tizard, Ian R. Veterinary immunology. Australian Veterinary Journal. 2011;48(2):550-551.
31. Ziv G, HEAVNER JE. Permeability of the blood-milk barrier to methylene blue in cows and goats. Journal of Veterinary Pharmacology \& Therapeutics.7(1):55-59.

32. Cadwell K. Crosstalk between autophagy and inflammatory signalling pathways: balancing defence and homeostasis. Nature reviews Immunology. 2016;16(11):661-675.

33. Luciani A, Festa BP, Chen Z, Devuyst O. Defective autophagy degradation and abnormal tight junction-associated signaling drive epithelial dysfunction in cystinosis. Autophagy. 2018;14(7):1157-1159.

34. Yeung YT, Aziz F, Guerrero-Castilla A, Arguelles S. Signaling Pathways in Inflammation and Anti-inflammatory Therapies. Curr Pharm Des. 2018;24(14):1449-1484

35. Delbridge LMD, Mellor KM, Taylor DJ, Gottlieb RA. Myocardial stress and autophagy: mechanisms and potential therapies. Nature reviews Cardiology. 2017;14(7):412-425

36. Filomeni G, De Zio D, Cecconi F. Oxidative stress and autophagy: the clash between damage and metabolic needs. Cell death and differentiation. 2015;22(3):377-388

37. Li L, Wan G, Han B, Zhang Z. Echinacoside alleviated LPS-induced cell apoptosis and inflammation in rat intestine epithelial cells by inhibiting the mTOR/STAT3 pathway. Biomed Pharmacother. 2018;104:622-628.

38. Yan L, Xu F, Dai CL. Relationship between epithelial-to-mesenchymal transition and the inflammatory microenvironment of hepatocellular carcinoma. J Exp Clin Cancer Res. 2018;37(1):203.

39. Sfanos KS, Yegnasubramanian $S$, Nelson WG, De Marzo AM. The inflammatory microenvironment and microbiome in prostate cancer development. Nat Rev Urol. 2018;15(1):11-24.

40. Smith M, Wilkinson S. ER homeostasis and autophagy. Essays Biochem. 2017;61(6):625-635

41. Zhang ZW, Li ZL, Yuan S. The Role of Secretory Autophagy in Zika Virus Transfer through the Placental Barrier. Front Cell Infect Microbiol. 2016;6:206.

42. Elshaer D, Begun J. The role of barrier function, autophagy, and cytokines in maintaining intestinal homeostasis. Semin Cell Dev Biol. 2017;61:51-59.

43. Dodson M, Redmann M, Rajasekaran NS, Darley-Usmar V, Zhang J. KEAP1-NRF2 signalling and autophagy in protection against oxidative and reductive proteotoxicity. Biochem I. 2015;469(3):347-355.

44. Jiang T, Harder B, Rojo de la Vega M, Wong PK, Chapman E, Zhang DD. p62 links autophagy and Nrf2 signaling. Free Radic Biol Med. 2015;88(Pt B):199-204.

45. Ngabire D, Kim GD. Autophagy and Inflammatory Response in the Tumor Microenvironment. Int J Mol Sci. 2017;18(9).

46. Marchev AS, Dimitrova PA, Burns AJ, Kostov RV, Dinkova-Kostova AT, Georgiev MI. Oxidative stress and chronic inflammation in osteoarthritis: can NRF2 counteract these partners in crime? Ann $N$ Y Acad Sci. 2017;1401(1):114-135

47. Loboda A, Damulewicz M, Pyza E, Jozkowicz A, Dulak J. Role of Nrf2/HO-1 system in development, oxidative stress response and diseases: an evolutionarily conserved mechanism. Cell Mol Life Sci. 2016;73(17):3221-3247.

48. Liu Q, Lv H, Wen Z, Ci X, Peng L. Isoliquiritigenin Activates Nuclear Factor Erythroid-2 Related Factor 2 to Suppress the NOD-Like Receptor Protein 3 Inflammasome and Inhibits the NF-kappaB Pathway in Macrophages and in Acute Lung Injury. Frontiers in immunology. 2017;8:1518

49. Nighot PK, Hu CA, Ma TY. Autophagy enhances intestinal epithelial tight junction barrier function by targeting claudin-2 protein degradation. I Biol Chem. 2015;290(11):7234-7246.

50. Chen $\mathrm{H}, \mathrm{Li} \mathrm{J}, \mathrm{Li} \mathrm{H}$, et al. Transcript profiling identifies dynamic gene expression patterns and an important role for Nrf2/Keap1 pathway in the developing mouse esophagus. PLoS One. 2012;7(5):e36504. 